\title{
A Comprehensive and Modular Stochastic Modeling Framework for the Variability-Aware Assessment of Signal Integrity in High-Speed Links
}

\author{
Yinghao Ye, Student Member, IEEE, Domenico Spina, Member, IEEE, Paolo Manfredi, Member, IEEE, \\ Dries Vande Ginste, Senior Member, IEEE, and Tom Dhaene, Senior Member, IEEE
}

\begin{abstract}
This paper presents a comprehensive and modular modeling framework for stochastic signal integrity analysis of complex high-speed links. Such systems are typically composed of passive linear networks and nonlinear, usually active, devices. The key idea of the proposed contribution is to express the signals at the ports of each of such system elements or subnetworks as a polynomial chaos expansion. This allows one to compute, for each block, equivalent deterministic models describing the stochastic variations of the network voltages and currents. Such models are synthesized into SPICE-compatible circuit equivalents, which are readily connected together and simulated in standard circuit simulators. Only a single circuit simulation of such an equivalent network is required to compute the pertinent statistical information of the entire system, without the need of running a large number of time-consuming electromagnetic-circuit cosimulations. The accuracy and efficiency of the proposed approach, which is applicable to a large class of complex circuits, are verified by performing signal integrity investigations of two interconnect examples.
\end{abstract}

Index Terms-Polynomial Chaos, nonlinear, variability analysis, passivity.

\section{INTRODUCTION}

$\mathbf{I}$ $\mathrm{N}$ recent years, a great deal of attention has been devoted to study the effects of geometrical and/or electrical parameter variations on the performance of various electronic circuits. Indeed, circuit performance is nowadays largely affected by the tolerances of the manufacturing process, due to the increasing integration and miniaturization. In this context, uncertainty quantification methods become a critical resource for the signal integrity (SI) assessment of high-speed designs. The Monte Carlo (MC) method is robust, accurate and easy to implement, and it is considered as the standard approach for variability analysis. However, its high computational cost often prohibits its application to the analysis of complex designs. Therefore, more efficient techniques for stochastic simulations are needed.

Many techniques based on the polynomial chaos (PC) method [1], [2] have been recently developed to tackle this problem [3]-[14], and are able to perform variability analysis with high accuracy and efficiency compared to MC-based

Manuscript received April 28, 2017; revised June 01, 2017. The work was supported by the Research Foundation - Flanders (FWO), of which Dr. Manfredi is a Postdoctoral Research Fellow.

Y. Ye, D. Spina, P. Manfredi, D. Vande Ginste, T. Dhaene are with IDLab, Department of Information Technology, Ghent University-imec, B9052 Ghent, Belgium, e-mail: \{yinghao.ye, domenico.spina, paolo.manfredi, dries.vandeginste, tom.dhaene $\} @$ ugent.be. approaches. Each method is tailored for a specific class of circuits, like lumped-element circuits [3], transmissionline circuits [4], [5], arbitrary passive linear systems [6][8], or nonlinear circuits [9]-[14]. However, a general and comprehensive modeling framework, capable of including heterogeneous stochastic components appears yet to be missing. Indeed, modern interconnect designs consist of a combination of circuit-level components, behavioral macromodels, and 3D structures. The present paper aims at covering the aforementioned gap by proposing a comprehensive and modular approach that allows the inclusion of any linear passive structure (possibly characterized by means of full-wave EM simulations) or nonlinear component (either at physical or behavioral level). The point of departure is the technique presented in [8], which was recently proposed to compute a stable and passive PC-based macromodel of a generic linear, passive and frequency-dependent stochastic system. It is a flexible and reliable modeling approach, but it is limited to passive linear systems.

In order to overcome this limitation, we integrate the technique proposed in [13], which can create a deterministic equivalent circuit model of any stochastic nonlinear component at both the physical or behavioral level, thus yielding a novel and powerful framework for the variability-aware SI analysis of complex high-speed circuit designs. The models for each linear and nonlinear subpart are computed separately, with the desired (or most convenient) level of modularity, and then connected together in a SPICE-type environment. Within the novel modeling framework, only a single time-domain simulation of the entire system is required to perform the variability analysis, thus avoiding expensive repeated (EM-)circuit (co)-simulations. As will be shown in Section III, complete interconnect links can as such be analyzed very efficiently.

The paper is organized as follows. Section II describes the novel proposed approach, while its validation is carried out in Section III by means of two pertinent numerical examples. Conclusions are drawn in Section IV.

\section{Stochastic Modeling of COMPlex Links}

Modern high-speed links consist of a combination of nonlinear, possibly active components (e.g., diodes, drivers and receivers) and passive linear structures (e.g., transmission lines, power distribution networks, vias, packages, etc.). While the former are defined by nonlinear relations between their 
port signals, which can be defined in different ways (for example analytical formulas or behavioral models), the latter are completely defined by their transfer function, which can be expressed in several forms (i.e., impedance, admittance or scattering representations) and may require time-consuming EM simulations to be determined.

In the following, we assume that the linear parts of the system under study depend on some geometrical or electrical random parameters collected in the vector $\boldsymbol{\xi}$, while the nonlinear elements are influenced by the vector of random variables $\hat{\boldsymbol{\xi}}$. As a result of this variability, the voltages and currents at each port of such linear and nonlinear components depend on both the random variables $\hat{\boldsymbol{\xi}}$ and $\boldsymbol{\xi}$, as illustrated in Fig. 1.

According to the PC theory, such port signals are represented as a truncated summation of basis functions with suitable coefficients:

$$
v(\hat{\boldsymbol{\xi}}, \boldsymbol{\xi}, t) \approx \sum_{i=0}^{M} v_{i}(t) \varphi_{i}(\hat{\boldsymbol{\xi}}, \boldsymbol{\xi})
$$

where $v(\hat{\boldsymbol{\xi}}, \boldsymbol{\xi}, t)$ denotes a generic voltage within the system (currents are treated in a similar fashion), the basis functions $\varphi_{i}(\hat{\boldsymbol{\xi}}, \boldsymbol{\xi})$ are orthogonal polynomials depending on the joint distribution of the random variables $\boldsymbol{\xi}$ and $\hat{\boldsymbol{\xi}}$, and $v_{i}(t)$ are the corresponding $M+1$ coefficients to be determined. The main feature of the PC expansion (1) is its efficient representation of the variability of the quantity under study. For example, stochastic moments, such as mean and variance, are readily obtained from the coefficients of (1) [1], [2]. Other statistical functions, like the probability density function (PDF) or the cumulative distribution function (CDF), can be calculated via the (inexpensive) sampling of (1).

The key idea of the proposed approach is to describe the stochastic variations of both the linear and nonlinear system subnetworks via suitable PC models, which will simultaneously account for all the random variables $\boldsymbol{\xi}$ and $\hat{\boldsymbol{\xi}}$. Specifically, the procedure foresees the computation of equivalent and deterministic circuit models describing the time-domain behavior of the PC coefficients of the port voltages and currents, starting from the original stochastic system, which are then connected together according to the original circuit topology. In the PC jargon, such models are usually referred to as "augmented" [8], since their number of ports is higher with respect to the corresponding stochastic elements. However, as the model is deterministic, a single transient circuit simulation (e.g., in SPICE) allows determining all the PC expansion coefficients in (1) and hence the desired statistical information.

At this point, it is important to stress two important features offered by the advocated modeling strategy to circuit designers:

- The equivalent models can be constructed with the desired level of modularity. For example, subparts that have already a circuit-level description can still be modeled using the approaches for lumped and distributed elements in [10] and [4], respectively. However, if the stochastic behavior of their inner voltages and currents is not of interest, they can be encompassed in a larger macromodel.

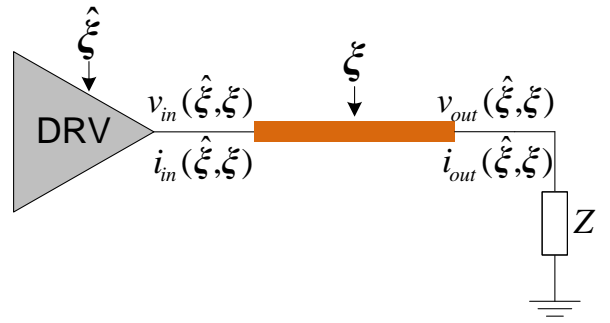

Fig. 1. Example of a link subject to stochastic variations affecting a (nonlinear) driver (DRV), a transmission line and a lumped termination.

- Circuit-EM co-simulations are avoided, as every block is synthesized into a SPICE-compatible equivalent circuit network.

The method adopted for the modeling of linear networks is detailed in Section II-A, while Section II-B addresses the modeling of the nonlinear components. For the sake of simplicity, in the remainder of this paper we will describe only the case of independent random variables, even though it is possible to extend the proposed method to the case of correlated random variables [1], [2]. Furthermore, only orthonormal PC basis functions will be considered. Please note that discussing the properties of the PC expansion is outside the scope of this contribution. The interested reader may consult [1]- [13] for an extensive reference to PC theory.

\section{A. Stochastic Modeling of General Passive Linear Systems}

When a generic linear system is subjected to stochastic effects caused by random variations of geometric or electrical parameters, here denoted by $\boldsymbol{\xi}$, the pertinent scattering matrix will be $\boldsymbol{\xi}$ dependent. If such a system is embedded in a network that is also affected by the random variables $\hat{\xi}$, the incident and reflected waves will in general depend on both $\boldsymbol{\xi}$ and $\hat{\xi}$ (see Fig. 1). The scattering relations can therefore be expressed as

$$
\boldsymbol{b}(\boldsymbol{\xi}, \hat{\boldsymbol{\xi}}, s)=\boldsymbol{S}(\boldsymbol{\xi}, s) \boldsymbol{a}(\boldsymbol{\xi}, \hat{\boldsymbol{\xi}}, s)
$$

where $S$ is the scattering parameter matrix of the stochastic linear system, $s$ is the Laplace variable, and $\boldsymbol{a} \in \mathbb{C}^{N \times 1}$ and $\boldsymbol{b} \in \mathbb{C}^{N \times 1}$ are the incident and reflected waves, respectively, with $N$ the number of ports of the system.

The starting point of the proposed method is the computation of a PC model of the system scattering parameters over a discrete set of frequency values $f_{l}$ for $l=1, \ldots, L$ (or the Laplace variable $s_{l}=j 2 \pi f_{l}$ ) as:

$$
\boldsymbol{S}\left(\boldsymbol{\xi}, s_{l}\right) \approx \sum_{i=0}^{M_{S}} \boldsymbol{S}_{i}\left(s_{l}\right) \varphi_{i}(\boldsymbol{\xi})
$$

where $M_{S}+1$ is the number of basis functions depending only on $\boldsymbol{\xi}$. Any non-intrusive PC-based approach can be used for this purpose: the PC coefficients in (3) can be obtained via linear regression-, numerical integration- [2], or stochastic testing (ST)-based techniques [14], for example. 
Now, it is easy to express (3) with respect to all the random variables considered $(\hat{\boldsymbol{\xi}}, \boldsymbol{\xi})$ as

$$
\boldsymbol{S}\left(\hat{\boldsymbol{\xi}}, \boldsymbol{\xi}, s_{l}\right) \approx \sum_{i=0}^{M} \boldsymbol{S}_{i}\left(s_{l}\right) \varphi_{i}(\hat{\boldsymbol{\xi}}, \boldsymbol{\xi})
$$

since $\varphi_{i}(\boldsymbol{\xi})$ for $i=0, \ldots, M_{S}$ are a subset of $\varphi_{i}(\hat{\boldsymbol{\xi}}, \boldsymbol{\xi})$ for $i=0, \ldots, M$, thanks to the property that, for independent random variables, the PC basis functions in (4) are the product combination of the basis functions for each random variable considered [1], [2]. Hence, all the PC coefficients in (4) for basis functions depending on $\hat{\xi}$ are zero.

Nevertheless, a full expression of the PC expansion in the form (4) is required to properly account for the additional variability introduced by the rest of the network.

Now, by following the approach presented in [8], it is possible to apply Galerkin projections (GP) [1] to (2) and describe the relationship between the PC coefficients of the incident and reflected waves as:

$$
\boldsymbol{b}_{P C}\left(s_{l}\right)=\boldsymbol{S}_{P C}\left(s_{l}\right) \boldsymbol{a}_{P C}\left(s_{l}\right) \quad \text { for } l=1, \ldots, L
$$

where the vectors $\boldsymbol{a}_{P C}, \boldsymbol{b}_{P C} \in \mathbb{C}^{(M+1) N \times 1}$ collect the (deterministic) PC coefficients of the corresponding incident and reflected wave, respectively, with $M+1$ the total number of the PC basis functions, whereas $\boldsymbol{S}_{P C} \in \mathbb{C}^{(M+1) N \times(M+1) N}$ is a deterministic matrix, obtained by suitable combination of the PC coefficients of the scattering matrix in (4).

Equation (5) describes a new system, whose port voltages and currents are the PC coefficients of those of the original stochastic system. Note that $\boldsymbol{S}_{P C}$ is $M+1$ times larger than the original system in terms of number of ports. Furthermore, the matrices $\boldsymbol{S}_{P C}\left(s_{l}\right)$ for $l=1, \ldots, L$ are sparse by construction [8], since in (4) some PC coefficients are zero, as discussed above. Finally, in [8] it is proven that $\boldsymbol{S}_{P C}$ can still be regarded as a scattering parameter matrix, now relating the PC coefficients of the incident and reflected waves: its passivity conditions are therefore the same as for deterministic scattering parameters.

At this point, a rational model of $\boldsymbol{S}_{P C}$ is built by means of the Vector Fitting (VF) technique [15], where a poleflipping scheme is used to enforce stability, while passivity assessment and enforcement are accomplished by using standard and robust techniques [16]. Then, it is straightforward to convert such a rational model into a corresponding state-space representation [15], which can be converted into an equivalent circuit via a suitable synthesis technique [17].

A key step in any modeling process is adopting a suitable error measure and error threshold. The maximum absolute model error (MER) between the elements of the scattering parameter augmented matrix $\boldsymbol{S}_{P C}^{i, j}$ and the corresponding VF model response $\boldsymbol{H}_{P C}^{i, j}$ in the entire frequency range is chosen in [8] to compute the desired rational model of $\boldsymbol{S}_{P C}$ :

$$
M E R=\max _{i, j, l}\left(\left|\boldsymbol{S}_{P C}^{i, j}\left(s_{l}\right)-\boldsymbol{H}_{P C}^{i, j}\left(s_{l}\right)\right|\right)
$$

for $i, j=1, \ldots,(M+1) N$ and $l=1, \ldots, L$. Note that in [8], the choice of the MER error threshold was left to the designer's expertise, by considering that modeling $\boldsymbol{S}_{P C}$ is similar to modeling a deterministic scattering parameter matrix. Here, a new criterion is provided based on the unique characteristics of the $\boldsymbol{S}_{P C}$ augmented system. Indeed, thanks to the properties of the GP method, it is easy to prove that all the non-null elements of the first $N \times(M+1) N$ or $(M+1) N \times N$ block of $\boldsymbol{S}_{P C}$ are, by construction, formed by the PC coefficients of the scattering parameter matrix in (4).

Since the first two stochastic moments, i.e., the mean $\mu$ and the standard deviation $\sigma$, can be analytically computed from these coefficients [1], [2] in the following the MER error threshold in (6) is chosen such that the differences:

$$
\left\{\begin{array}{l}
\operatorname{error}_{\text {mean }}\left(s_{l}\right)=\max \left(\left|\boldsymbol{\mu}\left(s_{l}\right)-\boldsymbol{\mu}_{H}\left(s_{l}\right)\right|\right) \\
\operatorname{error}_{\text {sigma }}\left(s_{l}\right)=\max \left(\left|\boldsymbol{\sigma}\left(s_{l}\right)-\boldsymbol{\sigma}_{H}\left(s_{l}\right)\right|\right)
\end{array}\right.
$$

are minimized, where $\boldsymbol{\mu}_{H}$ and $\boldsymbol{\sigma}_{H}$ are the mean and standard deviation computed by the VF model of $\boldsymbol{S}_{P C}$ for all the frequency samples considered $l=1, \ldots, L$. Hence, the MER error threshold in (6) is chosen based on the model capability of accurately estimating the stochastic moments (7).

Finally, in [8] it is proven that the PC coefficients of the incident and reflected waves at the $k$-th port of the system depend only on the corresponding voltage and current PC coefficients at the same port, as for the original deterministic system. This property allows one to easily connect the model of $\boldsymbol{S}_{P C}$ to the models of the other system subparts, as will be discussed in details in the following.

It is important to point out that the modeling approach outlined in this section readily applies to any general electrical (e.g., circuit or EM) system supporting a scattering representation like (2).

\section{B. Stochastic Modeling of Nonlinear Components}

Nonlinear components affected by random variables $\hat{\xi}$ are described by suitable and possibly dynamic relationships between the time-domain counterparts of the port voltages and currents, here denoted as

$$
\boldsymbol{i}(\boldsymbol{\xi}, \hat{\boldsymbol{\xi}}, t)=\mathcal{F}(\boldsymbol{v}(\boldsymbol{\xi}, \hat{\boldsymbol{\xi}}, t), t, \hat{\boldsymbol{\xi}})
$$

where vectors $\boldsymbol{v}(\boldsymbol{\xi}, \hat{\boldsymbol{\xi}}, t)$ and $\boldsymbol{i}(\boldsymbol{\xi}, \hat{\boldsymbol{\xi}}, t)$ collect all port voltages and currents, whereas $\mathcal{F}$ is understood to be a vectorized operator whose $k$-th row, $i_{k}=\mathcal{F}_{k}(\boldsymbol{v}, t, \hat{\boldsymbol{\xi}})$, describes the nonlinear relationship between the $k$-th port current and (all) port voltages. This can be either an explicit nonlinear function or an implicit characteristic imposed by a generic nonlinear component or subcircuit (e.g, diode, transistor, or behavioral model). As already mentioned for the linear components, the port voltages and currents are stochastic as a result of the variability in both the linear and nonlinear parts of the network, and $\mathcal{F}$ is subjected to stochastic effects, but only due to $\hat{\boldsymbol{\xi}}$.

Replacing the port voltages and currents in (8) with their PC expansions, and enforcing the resulting equation to hold for $M+1$ values $\left\{\boldsymbol{\xi}_{m}, \hat{\boldsymbol{\xi}}_{m}\right\}_{m=0}^{M}$ of the random variables, predetermined via the ST algorithm [9], yields a deterministic 
system of $M+1$ equations in the voltage and current $\mathrm{PC}$ coefficients. For example, for the current at the $k$-th port:

$$
\begin{aligned}
& a_{00} i_{k 0}(t)+\ldots+a_{0 M} i_{k M}(t) \\
& =\mathcal{F}_{k}\left(a_{00} \boldsymbol{v}_{0}(t)+\ldots+a_{0 M} \boldsymbol{v}_{M}(t), t, \hat{\boldsymbol{\xi}}_{0}\right) \\
& a_{M 0} i_{k 0}(t)+\ldots+a_{M M} i_{k M}(t) \\
& =\mathcal{F}_{k}\left(a_{M 0} \boldsymbol{v}_{0}(t)+\ldots+a_{M M} \boldsymbol{v}_{M}(t), t, \hat{\boldsymbol{\xi}}_{M}\right)
\end{aligned}
$$

where $a_{m i}$ are mere scalar coefficients obtained by evaluating the $i$-th PC basis function at the $m$-th ST point:

$$
a_{m i}=\varphi_{i}\left(\hat{\boldsymbol{\xi}}_{m}, \boldsymbol{\xi}_{m}\right)
$$

It is important to note that, while the nonlinear characteristic $\mathcal{F}_{k}$ in (9) itself depends solely on the ST points $\hat{\boldsymbol{\xi}}_{m}$, its inputs depend also on the points $\boldsymbol{\xi}_{m}$. In general, even for deterministic nonlinear elements, the voltage and current PC coefficients are coupled, as opposed to the case of deterministic linear components [10], [13].

From (9), an explicit relation for the coefficients of the port current is obtained by inverting the system (9) in matrix form:

$$
\begin{aligned}
{\left[\begin{array}{c}
i_{k 0}(t) \\
\vdots \\
i_{k M}(t)
\end{array}\right] } & =\left[\begin{array}{ccc}
a_{00} & \ldots & a_{0 M} \\
\vdots & \ddots & \vdots \\
a_{M 0} & \ldots & a_{M M}
\end{array}\right]^{-1} \\
\times & {\left[\begin{array}{c}
\mathcal{F}_{k}\left(a_{00} \boldsymbol{v}_{0}(t)+\ldots+a_{0 M} \boldsymbol{v}_{M}(t), t, \hat{\boldsymbol{\xi}}_{0}\right) \\
\vdots \\
\mathcal{F}_{k}\left(a_{M 0} \boldsymbol{v}_{0}(t)+\ldots+a_{M M} \boldsymbol{v}_{M}(t), t, \hat{\boldsymbol{\xi}}_{M}\right)
\end{array}\right] }
\end{aligned}
$$

The system (11) has a SPICE-compatible circuit interpretation, as described in [13]. It can be inferred from the vector term in the r.h.s. of (11) that such an equivalent circuit requires $M+1$ deterministic replicas of the nonlinear component, for which the stochastic parameters are evaluated as defined by the corresponding ST points. The general topology of the circuit model is, however, independent of the specific type of nonlinear element, as long as it has a description of the form (8).

\section{Overall Network Model}

The augmented deterministic models described in Sections II-A and II-B must be suitably connected together to produce the complete model of the stochastic network under study. This is done in accordance with the original network topology. For example, Fig. 2(a) shows the connection of a 2-port nonlinear device to a 2-port linear network. The two components are subjected to stochastic effects due to two random variables $\hat{\xi}$ and $\xi$, respectively. For the sake of illustration, we consider a first-order PC expansion, leading to a total of three basis functions. The extension to systems with a higher number of ports and/or of PC basis functions is straightforward.

The SPICE-compatible circuit model for the nonlinear part is shown in Fig. 2(b). It includes three instances $\mathcal{F}\left(\hat{\xi}_{0}\right)$, $\mathcal{F}\left(\hat{\xi}_{1}\right), \mathcal{F}\left(\hat{\xi}_{2}\right)$ of the original stochastic nonlinear component, in which the random parameter $\hat{\xi}$ is set to values $\hat{\xi}_{0}, \hat{\xi}_{1}$ and $\hat{\xi}_{2}$, respectively. The required voltages at the ports of these instances are produced by means of controlled voltage sources,

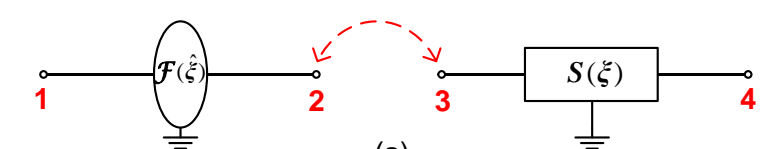

(a)

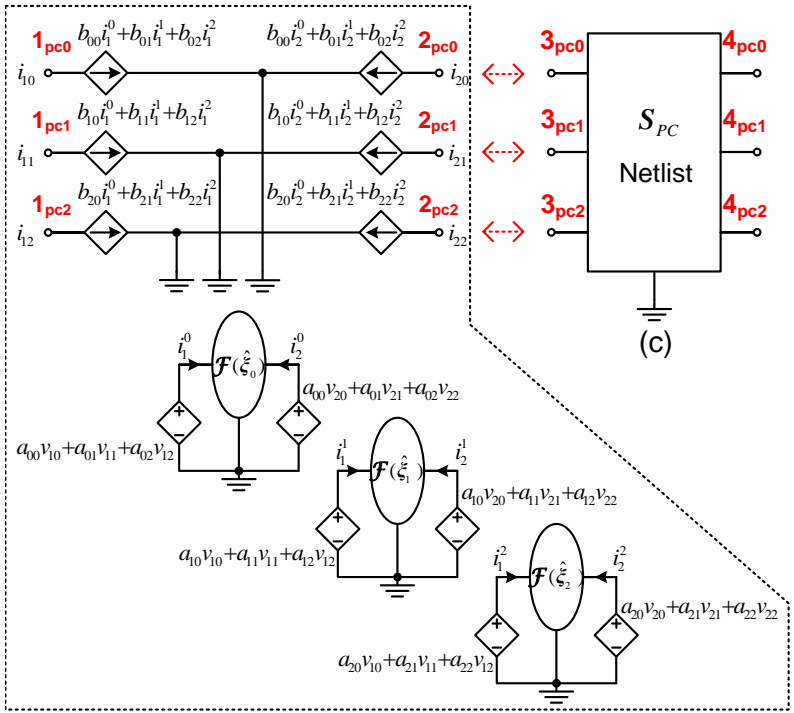

(b)

Fig. 2. (a) Connected nonlinear and linear systems subjected to stochastic effects; (b) and (c) Deterministic equivalent models of the stochastic nonlinear and linear systems.

with coefficients as in (10). Analogously, the correct expression of the currents in the main circuit (11) is reproduced by means of controlled current sources, with coefficients

$$
\left[\begin{array}{lll}
b_{00} & b_{01} & b_{02} \\
b_{10} & b_{11} & b_{12} \\
b_{20} & b_{21} & b_{22}
\end{array}\right]=\left[\begin{array}{lll}
a_{00} & a_{01} & a_{02} \\
a_{10} & a_{11} & a_{12} \\
a_{20} & a_{21} & a_{22}
\end{array}\right]^{-1}
$$

The interested readers may consult [13] for more detailed information about this model.

The model for the linear system in Fig. 2(c) is synthesized into an equivalent SPICE netlist consisting of lumped elements, which suitably describes the augmented system $\boldsymbol{S}_{P C}$ that is obtained as described in Section II-A. It is clear that each port of the original systems is now described by three corresponding ports in the augmented models, one for each PC coefficient of the port voltages and currents. The connectivity between the ports remains consistent for the augmented models, as shown in Fig. 2.

The resulting netlist can be readily simulated in a SPICEtype circuit simulator. A single time-domain simulation simultaneously yields all the PC coefficients of the port voltages and currents, which in turn provide comprehensive statistical information. The outlined modeling strategy offers circuit designers a modular and powerful tool allowing for the stochastic analysis of a system consisting of an arbitrary combination of passive linear systems and nonlinear components, each possibly affected by variability. The flowchart of the proposed modeling framework is shown in Fig. 3.

\section{ApPlications And Numerical Results}

This section discusses two application examples, concerning the SI analysis of i) an interconnect tree and ii) a nonuniform 


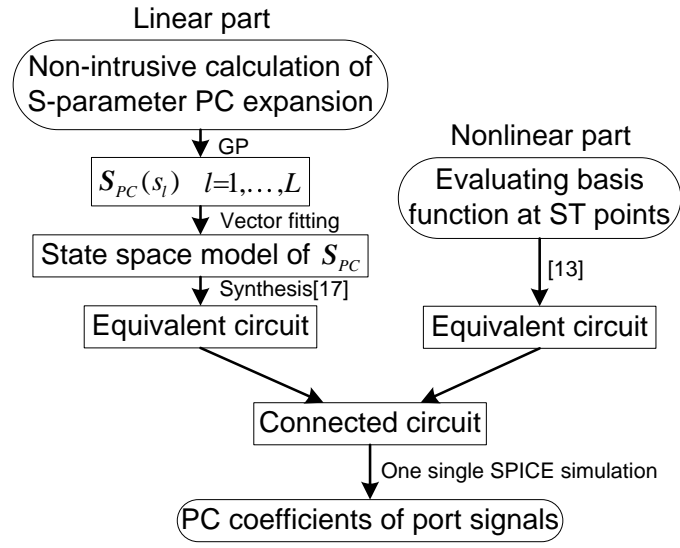

Fig. 3. Flowchart of the proposed statistical simulation framework.

differential link. All the time-domain simulations are carried out with HSPICE on a computer with an Intel(R) Core(TM) I3 Processor running at $2.93 \mathrm{GHz}$ and $8 \mathrm{~GB}$ of RAM.

\section{A. Interconnect Tree}

The first application example concerns the interconnect tree depicted in Fig. 4, consisting of both lumped interconnections and microstrip segments, whose cross-section is also indicated in the figure. The tree is driven at the input port by a behavioral macromodel of an I/O transceiver of a $512-\mathrm{Mb}$ Flash memory chip, which produces a pulse with a risetime of $1 \mathrm{~ns}$. The behavioral macromodel is obtained with the techniques in [18]-[20]. Each of the four output ports is terminated by a pair of diodes with saturation current $I_{S}=50 \mathrm{pA}$, series resistance $R_{S}=5 \Omega$, and junction capacitance $C_{J O}=3 \mathrm{pF}$. Not shown in Fig. 4 is the RL power supply network for the driver, with supply voltage $V_{D D}=1.8 \mathrm{~V}, R=10 \mathrm{~m} \Omega$ and $L=2 \mathrm{nH}$.

The thickness and relative permittivity of the microstrip substrate are assumed to be affected by process variations and therefore to vary according to a Gaussian distribution with a relative standard deviation of $5 \%$ of their nominal values.

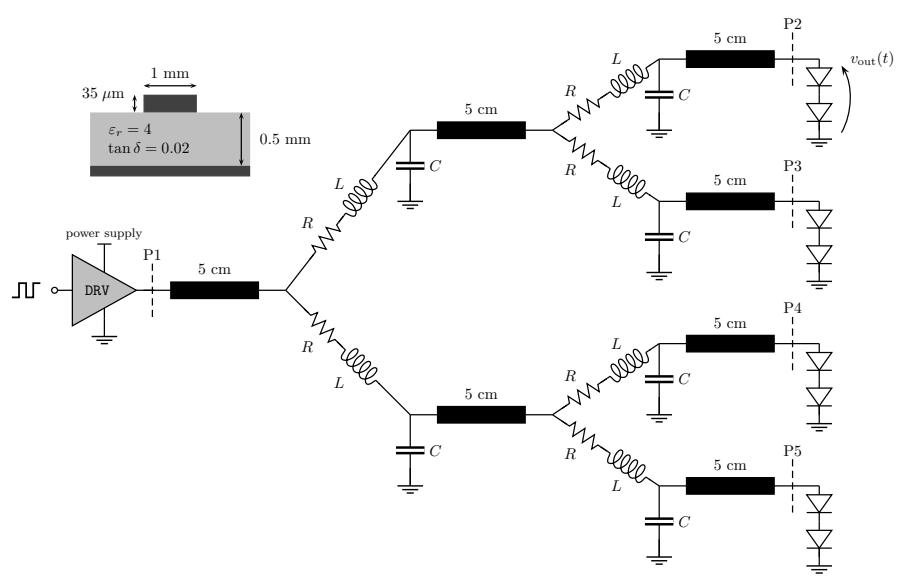

Fig. 4. Schematic of the interconnect tree, composed of seven identical lossy microstrip transmission lines, driven by a nonlinear I/O transceiver and terminated with diodes.
Moreover, the operating temperature at the receiver side is considered as an additional Gaussian random variable with nominal value $60^{\circ} \mathrm{C}$ and a standard deviation of $3^{\circ} \mathrm{C}$. This temperature fluctuation affects the operation of the diodes. Hence, there are three independent random parameters in the entire network, and a second-order PC expansion is used to describe the variations of the port signals, leading to a total of 10 PC basis functions. Now, in order to stress the versatility of the advocated modeling strategy, we consider the following alternative approaches to model the linear part of the network, based on the procedure outlined in Section II-A:

- the transmission-line elements are modeled as stochastic 2-port devices, the modeling of the remaining deterministic RLC elements being trivial, as discussed later;

- the entire linear part is modeled as a 5-port stochastic network.

In either cases, the linear models are complemented with the pertinent models of the nonlinear (deterministic) driver and (stochastic) diodes to obtain the equivalent PC-based model of the overall network. These two scenarios allow us not only to highlight the modularity of the proposed technique, but also to assess its performance for different modeling choices.

The scattering parameters of both the 5-port linear subnetwork and the 2-port transmission-line elements are evaluated at 561 frequency points in the range [0 - 3.5] GHz, 188 of which are used to build the corresponding $\boldsymbol{S}_{P C}$ models. The remaining samples are used for validation, thus verifying the model accuracy at frequencies that were not used to create the model.

Note that, as a result of the multiplication of the original port numbers by the number of PC basis functions, the model for the entire interconnection has 50 ports, while the model of each transmission-line segment has only 20 ports (see Section II-A). However, in the latter case the models for each transmission line need to be properly connected between each other in order to describe the variation of the entire interconnection. Therefore, an equivalent model of the lumped RLC elements must be generated as well. Yet, since these lumped elements are in this case deterministic, the corresponding model matrix is block diagonal, which means the transmission line models are interconnected by suitable replicas of the original RLC elements in Fig. 4 [4].

At this point, two alternative deterministic equivalent models describing the statistical variations of the interconnect in Fig. 4 have been computed, one starting from the scattering parameters of the entire interconnection and one from the scattering parameters of the single transmission-line elements. In the following, the results obtained using these two models, synthesized into SPICE-compatible networks, are denoted with "Simu $u_{I}$ " and "Simu $u_{T}$ ", respectively.

Now, the performance of the proposed models is compared not only against the results of a MC analysis, but also with the technique in [4]. Indeed, [4] offers an accurate and efficient reference approach, which can however be used only as long as the transmission lines are uniform, as is the case for the network of Fig. 4. On the contrary, the new proposed modeling strategy is applicable to any arbitrary line layout, as will be shown in the second application example. 


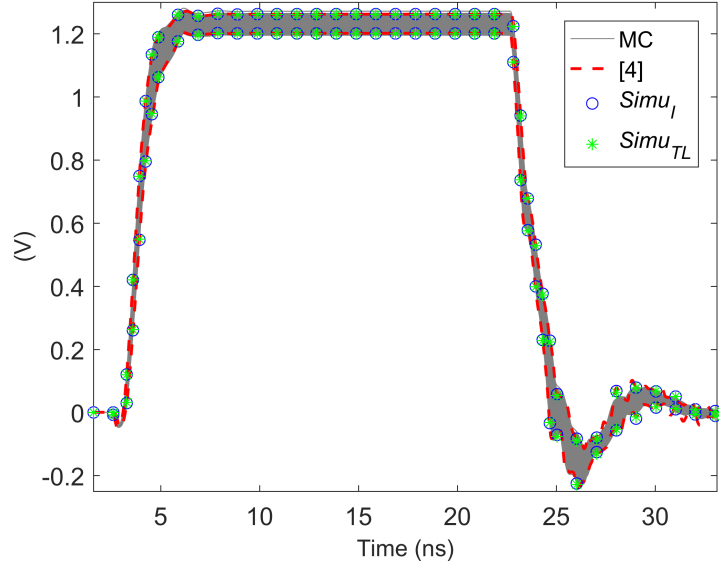

Fig. 5. Statistical analysis of the output voltage $v_{\text {out }}$ in the circuit of Fig. 4. The thin gray lines represent 10000 samples from MC analysis; the dashed red lines, and markers $\circ, *$ represent the $0.05 \%-99.95 \%$ quantiles obtained with the technique in [4], $S i m u_{I}$, and $S i m u_{T L}$, respectively.

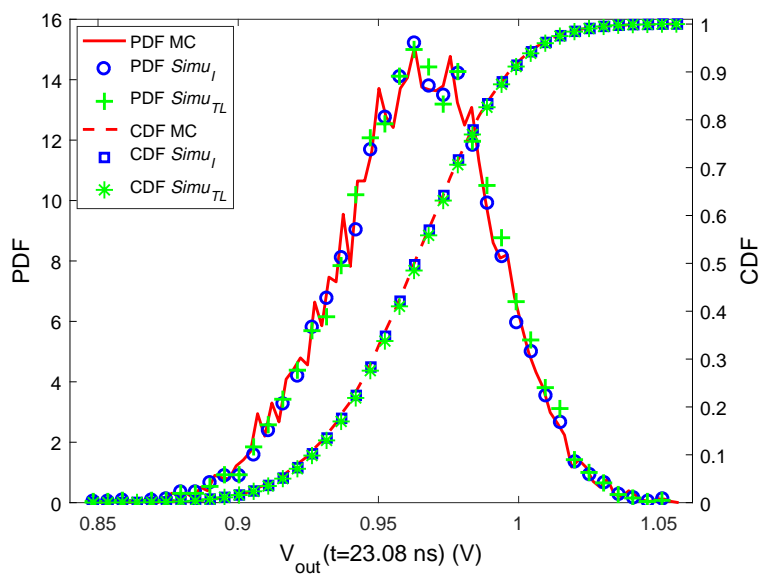

Fig. 6. PDF and CDF of the output voltage. The solid line, and markers $\circ$, + represent the PDF calculated with the MC method, Simu ${ }_{I}$, and $S_{i m u}{ }_{T}$, respectively; the dashed line and markers $\square, *$ represent the CDF calculated from the MC, Simu, , and $S i m u_{T L}$, respectively.

Fig. 5 shows the statistical behavior of the voltage $v_{\text {out }}$ at any (due to the structure symmetry) of the output ports P2P5 of the interconnect tree of Fig. 4. The thin gray curves are a superposition of 10000 random voltages from the MC analysis, which give a qualitative overview of the spread due to the variability. The dashed red lines and markers $\circ, *$ indicate the $0.05 \%$ and $99.95 \%$ quantiles estimated with the technique in [4], $S_{S T u}$ and $S i m u_{T L}$, respectively.

The PDF and CDF can also be obtained from the PC results. For example, Fig. 6 shows the PDF and CDF of the output voltage at the time instant $t=23.08 \mathrm{~ns}$, obtained by means of the MC method, as well as with Simu and $\operatorname{Simu}_{T L}$. The good agreement between these techniques demonstrates the accuracy and flexibility of the proposed approach. It is interesting to note that these results are obtained by using PC models of the same order as for the technique in [4], which relies however on a PC expansion of the transmission-line RLGC parameters rather than of the scattering parameters.

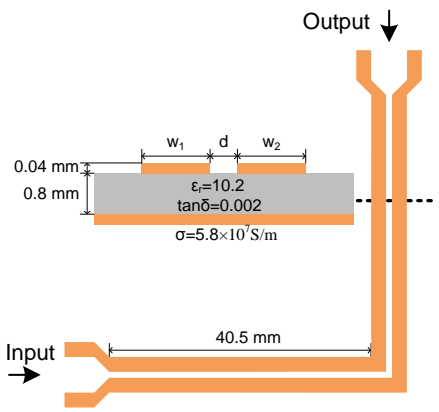

Fig. 7. Layout and cross-section of the bent microstrip differential line.

The simulation of $10000 \mathrm{MC}$ samples for the circuit under study required $41248 \mathrm{~s}$, while $S i m u_{I}, S_{i m u}$, and the technique in [4] required $139 \mathrm{~s}, 91 \mathrm{~s}$, and $45 \mathrm{~s}$, respectively. Hence, the new technique offers a great efficiency with respect to the MC analysis, achieving a speed-up factor of $297 \times$ $\left(S_{i m u}\right)$ and $453 \times\left(\operatorname{Simu}_{T L}\right)$. This not only demonstrates the flexibility of the proposed technique, but also shows an alternative and more efficient way to deal with complex systems by modeling their stochastic subnetworks.

On the other hand, it should be noted that the technique in [4] offers an even superior efficiency because it directly models the RLGC parameters of the transmission lines. It is well known that VF is not very efficient for modeling electricallylong interconnects, which may lead to an increase of the model complexity with consequent reduction of simulation efficiency as the interconnect length and/or port number is increased. Hence, a lower computational efficiency is to be expected when simulating a network that consists of circuit components only. Nevertheless, as already mentioned, the proposed approach has the considerable advantage of being more general and applicable to any passive linear system, since it is based on a scattering representation.

\section{B. Nonuniform Differential Line}

Coupled microstrip lines are widely used in modern highspeed digital circuits to transmit differential signals across boards, with higher EM immunity against conducted and radiated noise, lower interference and lower crosstalk. When using such differential line circuits, it is important to minimize the risk of differential-to-common mode conversion. However, layout constraints due to large-scale integration and miniaturization often make line bends unavoidable, which gives rise to such a mode conversion, and lead to a reduction in the spacing between the lines, which can exacerbate the impact of manufacturing tolerances, possibly leading to severe signal degradation. Therefore, it is important to quantify the effects of geometrical parameter variations on the SI in the early-stage design of the system.

As an example, the differential microstrip interconnect illustrated in Fig. 7 is analyzed in this section. The line exhibits a larger spacing between the traces at the terminal sections, which is reduced in the central part to achieve lower area occupation and lower mode conversion [21], and a 90-degree bend in the middle. As shown in Fig. 8, this nonuniform 


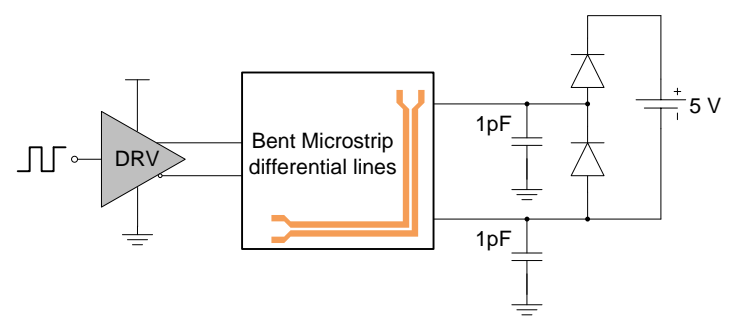

Fig. 8. Schematic of the complete differential link with driver and terminations.

coupled line is differentially driven at the input ports, and it is terminated with clamp diodes to limit voltage overand under-shoots at the receiver side. The driver is implemented using behavioral macromodels of a Texas Instruments transceiver (model name SN74ALVCH16973, power supply voltage $V_{D D}=2.5 \mathrm{~V}$ ). It produces a pseudo-random sequence of 1000 bits, with a bit time of $3 \mathrm{~ns}$ and a Gaussian jitter having a standard deviation of $0.15 \mathrm{~ns}$. The diodes have saturation current $I_{S}=50 \mathrm{pA}$, series resitance $R_{S}=1 \Omega$ and junction capacitance $C_{J O}=1 \mathrm{pF}$.

The values of the geometrical and electrical parameters of the microstrip line are shown in Fig. 7. In addition, the trace widths $w_{1}$ and $w_{2}$ are assumed to be independent Gaussian random variables, having a nominal value $0.625 \mathrm{~mm}$ and a relative standard deviation of 5\%. It is important to note that, since this interconnection must be studied by means of full-wave electromagnetic simulators and is terminated with nonlinear devices, its stochastic analysis cannot be addressed by previously proposed techniques.

The scattering matrices of the differential microstrip line are evaluated in $\mathrm{ADS}^{1}$ at 152 frequency points in the frequency range $[0-5] \mathrm{GHz}$ : half of them is used to build the desired model, the rest is used for validation. By following the procedure described in Section II-A, the augmented $\boldsymbol{S}_{P C}$ matrix is computed for each frequency sample starting from a PC expansion of order two, leading to six PC basis functions. Next, the equivalent state-space model is computed by means of the VF algorithm and then synthesized into a SPICE-compatible equivalent circuit. This equivalent circuit is complemented with the models for the driver and terminations, which are properly interconnected as discussed in Section II.

At this point, one transient SPICE simulation allows the designer to estimate the PC coefficients of the port signals, from which stochastic information pertaining the common and differential mode voltages are readily calculated.

A similar strategy is also adopted to run the comparative MC analysis. The scattering parameters of the stochastic system are evaluated for 10000 samples of the two random parameters $w_{1}, w_{2}$. For each sample, a stable and passive model is obtained by means of VF and then, via the same synthesis technique [17], converted into an equivalent circuit that is simulated in SPICE.

Figure 9 shows the probability of the common mode voltage levels, arising from both the mismatch in line length between

${ }^{1}$ Advanced Design System (ADS), Keysight Technologies, Santa Rosa, CA.
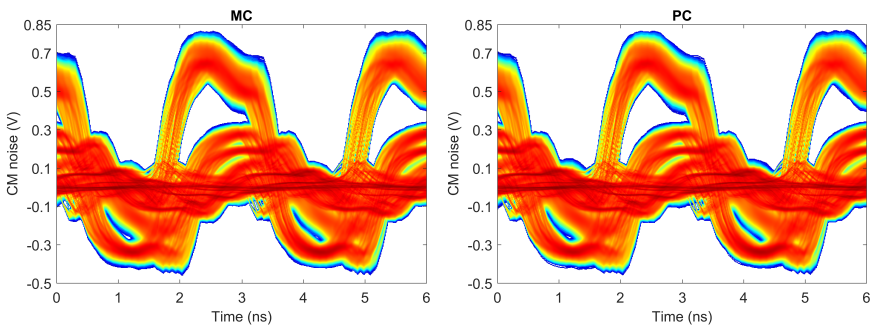

Fig. 9. Probability of common mode (CM) voltage levels in a two-bit time window calculated from the results of MC (left) and PC-based (right) analyses.

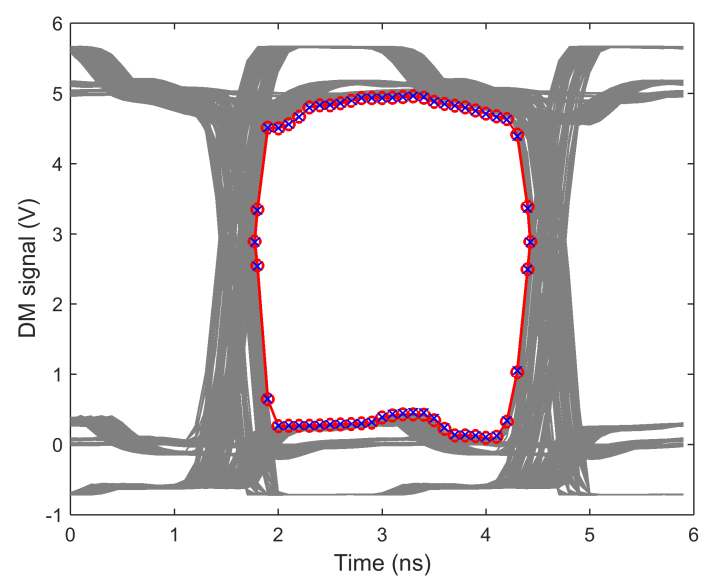

Fig. 10. Eye diagram of the differential model (DM) signal with inclusion of the eye opening profile computed with a $99 \%$ confidence level. Markers $\times$ and $\circ$ indicate the mask obtained from the MC samples and via the proposed technique, respectively.

the two traces [21] and the asymmetry introduced by the stochastic variations of the cross-sectional geometry [22], over a two-bit span resulting from the superposition of 10000 MC simulations for the entire 1000-bit input stream. The results from MC and PC-based analysis, the latter obtained by inexpensive sampling of the PC expansion (1), are compared side by side showing again excellent agreement.

Another important analysis for SI investigation is the prediction of the eye diagram. Fig. 10 shows the eye diagram of the output differential mode (DM) voltage for 10000 MC simulations of the bitstream transmission. In addition, markers $\times, \circ$ indicate the profile of the eye opening calculated with a $99 \%$ confidence level, which means there is a $1 \%$ probability that the stochastic differential mode response lies inside this mask.

Finally, Fig. 11 shows the probability distribution of the differential mode voltage obtained from both the MC (left panel) and PC (right panel) results, whereas Fig. 12 compares the PDF and CDF of the eye height calculated with the two methods. An excellent agreement between MC and the proposed PC-based technique is once again established.

As far as the computational times are concerned, the MC analysis based on 10000 samples required $5 \mathrm{~d} 19 \mathrm{~h} 44 \mathrm{~m}$ for the S-parameter extractions, $1 \mathrm{~h} 1 \mathrm{~m}$ for 10000 circuit models building and $22 \mathrm{~d} 14 \mathrm{~h} 49 \mathrm{~m}$ for the time-domain simulations. In contrast, the new proposed technique required $20 \mathrm{~m} 58 \mathrm{~s}$, 

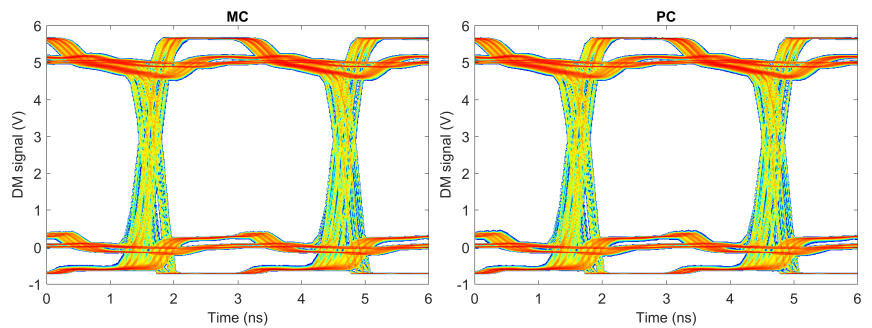

Fig. 11. Probability of differential mode (DM) voltage levels calculated from the results of MC (left) and PC-based (right) analyses.

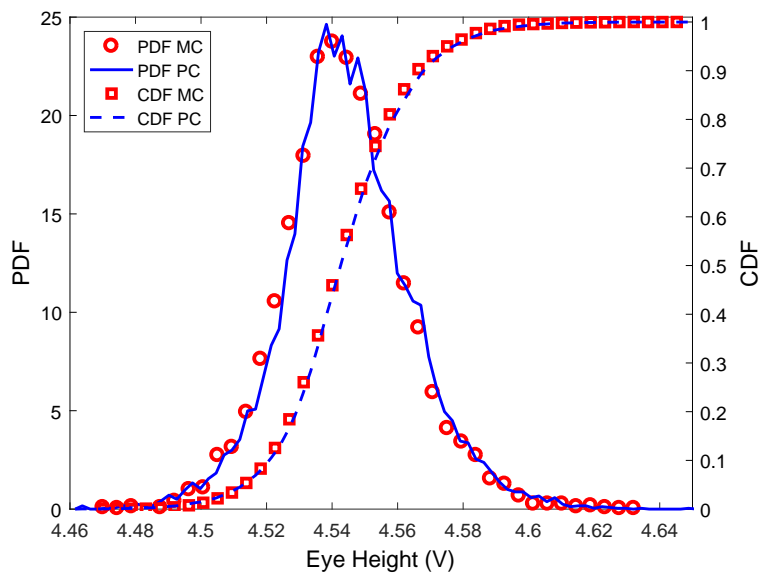

Fig. 12. PDF (full line) and CDF (dashed line) of the eye height computed using the new proposed technique. Markers $\circ$ and $\square$ indicate the same quantities computed with the MC method.

$9.4 \mathrm{~s}$ and $1 \mathrm{~h} 21 \mathrm{~m}$ for these three phases, respectively, thus achieving a speed-up of about $400 \times$ for each phase.

\section{CONCLUSION}

The efficient stochastic SI analysis of complex circuits including both passive linear subnetworks and nonlinear components is addressed in this paper. The proposed framework is based on representing the stochastic port voltages and currents of each component or subsystem as PC expansions. Deterministic augmented models are computed that describe the relationship between the pertinent PC coefficients. Such augmented models are synthesized into equivalent circuits, which are properly interconnected and solved with a single SPICE simulation to retrieve the time-domain behavior of the PC coefficients. From these PC coefficients, relevant statistical information about SI is obtained. The proposed method is modular and applicable to a broad range of systems composed by different elements (lumped, distributed, passive and active ones), thereby significantly expanding the scope of applicability of existing PC-based techniques. The accuracy, efficiency and flexibility of the modeling framework are illustrated by means of relevant numerical examples concerning SI investigations of digital interconnects.

\section{ACKNOWLEDGMENT}

The authors wish to express their gratitude to Prof. Igor Stievano, Politecnico di Torino, for providing the behavioral models used in the application examples.

\section{REFERENCES}

[1] D. Xiu and G.M. Karniadakis, "The Wiener-Askey polynomial chaos for stochastic differential equations," SIAM J. Sci. Comput., vol. 24, no. 2, pp. 619-644, Apr. 2002.

[2] M. S. Eldred, "Recent advance in non-intrusive polynomial-chaos and stochastic collocation methods for uncertainty analysis and design," in Proc. 50th AIAA/ASME/ASCE/AHS/ASC Struct., Structural Dynam., Mat. Conf., AIAA-2009-2274, Palm Springs, California, May 2009.

[3] K. Strunz and Q. Su, "Stochastic formulation of SPICE-type electronic circuit simulation with polynomial chaos," ACM Trans. Model. Comput. Simul., vol. 18, no. 4, pp. 15:1-15:23, Sep. 2008.

[4] P. Manfredi, D. Vande Ginste, D. De Zutter, and F. G. Canavero, "Uncertainty assessment of lossy and dispersive lines in SPICE-type environments," IEEE Trans. Compon., Packag., Manuf. Technol., vol. 3, no. 7, pp. 1252-1258, Jul. 2013.

[5] X. Chen, J. E. Schutt-Aine, and A. C. Cangellaris, "Stochastic LIM for transient simulations of printed circuit board transmission lines with uncertainties," in Proc. IEEE 64th Electron. Compon. Technol. Conf., Las Vegas, USA, May 2016.

[6] D. Spina, F. Ferranti, G. Antonini, T. Dhaene and L. Knockaert, "Efficient variability analysis of electromagnetic systems via polynomial chaos and model order reduction," IEEE Trans. Compon., Packag., Manuf. Technol., vol. 4, no. 6, pp. 1038-1051, Jun. 2014.

[7] D. Spina, F. Ferranti, T. Dhaene, L. Knockaert, and G. Antonini, "Polynomial chaos-based macromodeling of multiport systems using an input-output approach," Int. J. Numer. Model., vol. 28, no. 5, pp. 562581, Sep./Oct. 2015.

[8] D. Spina, T. Dhaene, L. Knockaert and G. Antonini, "Polynomial chaosbased macromodeling of general linear multiport systems for timedomain analysis," IEEE Trans. Microw. Theory Techn., vol. 65, no. 5, pp. 1422-1433, May 2017.

[9] Z. Zhang, T. A. El-Moselhy, I. M. Elfadel and L. Daniel, "Stochastic testing method for transistor-level uncertainty quantification based on generalized polynomial chaos," IEEE Trans. Comput.-Aided Design Integr. Circuits Syst, vol. 32, no. 10, pp. 1533-1545, Oct. 2013.

[10] P. Manfredi, D. Vande Ginste, D. De Zutter, and F. G. Canavero, "Stochastic Modeling of Nonlinear Circuits via SPICE-Compatible Spectral Equivalents," IEEE Trans. Circuits Syst. I, vol. 61, no. 7, pp. 2057-2065, Jul. 2014.

[11] M. R. Rufuie, E. Gad, M. Nakhla and R. Achar, "Generalized hermite polynomial chaos for variability analysis of macromodels embedded in nonlinear circuits," IEEE Trans. Compon., Packag., Manuf. Technol., vol. 4, no. 4, pp. 673-684, Apr. 2014.

[12] Z. Zhang, T. A. El-Moselhy, I. M. Elfadel and L. Daniel, "Calculation of generalized polynomial-chaos basis functions and Gauss quadrature rules in hierarchical uncertainty quantification," IEEE Trans. Comput.Aided Design Integr. Circuits Syst, vol. 33, no. 5, pp. 728-740, May 2014.

[13] P. Manfredi and F. G. Canavero, "Efficient statistical simulation of microwave devices via stochastic testing-based circuit equivalents of nonlinear components," IEEE Trans. Microw. Theory Techn., vol. 63, no. 5, pp. 1502-1511, May 2015.

[14] P. Manfredi, D. Vande Ginste, D. De Zutter, and F. G. Canavero, "Generalized decoupled polynomial chaos for nonlinear circuits with many random parameters," IEEE Microw. Wireless Compon. Lett., vol. 25, no. 8, pp. 505-507, Aug. 2015.

[15] B. Gustavsen and A. Semlyen, "Rational approximation of frequency domain responses by vector fitting," IEEE Trans. Power Del., vol. 14, no. 3, pp. 1052-1061, Jul. 1999.

[16] D. Deschrijver, and T. Dhaene, "Fast passivity enforcement of Sparameter macromodels by pole perturbation," IEEE Trans. Microw. Theory Techn., vol. 57, no. 3, pp. 620-626, Mar. 2009.

[17] R. Neumayer, F. Haslinger, A. Stelzer and R. Weigel, "Synthesis of SPICE-compatible broadband electrical models from n-port scattering parameter data," in Proc. IEEE Int. Symp. on Electromagnetic Compatibility (EMC), Minneapolis, USA, Aug. 2002.

[18] I. S. Stievano, I. A. Maio, and F. G. Canavero, "M $\pi \log$, macromodeling via parametric identification of logic gates," IEEE Trans. Adv. Packag., vol. 27, no. 1, pp. 15-23, Feb. 2004.

[19] G. Signorini, C. Siviero, S. Grivet-Talocia, and I. S. Stievano, "Macromodeling of I/O buffers via compressed tensor representations and rational approximations," IEEE Trans. Compon., Packag., Manuf. Technol., vol. 6 , no. 10, pp. 1522-1534, Oct. 2016. 
[20] G. Signorini, C. Siviero, M. Telescu, and I. S. Stievano, "Present and future of I/O-buffer behavioral macromodels," IEEE Electrmagn. Compat., vol. 5, no. 3, pp. 79-85, Third Quarter 2016.

[21] C. Gazda, D. Vande Ginste, H. Rogier, R. B. Wu, and D. De Zutter, "A wideband common-mode suppression filter for bend discontinuities in differential signaling using tightly coupled microstrips," IEEE Trans. Adv. Packag., vol. 33, no. 4, pp. 969-978, Nov. 2010.

[22] F. Grassi, Y. Yang, X. Wu, G. Spadacini, and S. A. Pignari, "On mode conversion in geometrically unbalanced differential lines and its analogy with crosstalk," IEEE Trans. Electromagn. Compat., vol. 57, no. 2, pp. 283-291, Apr. 2015. 